\title{
Information Rates of Active Propagation in Microchannel Molecular Communication
}

\author{
Nariman Farsad ${ }^{1}$, Andrew W. Eckford ${ }^{1}$, Satoshi Hiyama ${ }^{2}$, and Yuki Moritani ${ }^{2}$ \\ 1 Dept. of Computer Science and Engineering, York University \\ 4700 Keele Street, Toronto, ON, Canada M3J 1P3 \\ nariman@cse. yorku.ca, aeckford@yorku.ca \\ 2 Research Laboratories, NTT DOCOMO Inc., Yokosuka, Kanagawa, Japan \\ hiyama@nttdocomo.co.jp, moritani@nttdocomo.co.jp
}

\begin{abstract}
Molecular communication is a promising technique for microchannel systems. In this paper, various microchannel molecular communication schemes are simulated and analyzed using information theory, including molecular motors and Brownian motion with drift. Results suggest Brownian motion with drift can deliver excellent performance, depending on the drift velocity.
\end{abstract}

Key words: Molecular communication, microchannels, information theory.

\section{Introduction}

Molecular communication [1] is a new field of communication where instead of electric currents or electromagnetic waves, patterns of molecules are used to transfer information from a source (transmitter) to a destination (receiver). In this paper, we consider propagation media that are confined fluid environments with small dimensions in the order of micrometers, known as a microchannel. Molecular communication is a promising technology for passing control and information messages in microchannel and lab-on-chip systems [2].

In this paper we focus on the communication-theoretic and informationtheoretic aspects of molecular communication. Notable works in this direction include a general formulation of molecular communication as a timing channel under Brownian motion [3, 4], an analysis of information transfer rates using molecular motors [5, 6], mathematical channel models for continuous diffusion [7], and a simple model comparing the achievable information rates of passive transport using Brownian motion to that of active transport using microtubule filaments moving over a molecular motor track [8].

Our main contribution in this paper is to compare the mutual information of molecular communication systems in microchannels. Mutual information is a key parameter of a communication system, as it is the highest rate at which information can be reliably transmitted [9]. This paper extends our previous work in [8], with the following two novel aspects. Firstly, and more importantly, 
we focus primarily on active forms of propagation in this paper: molecular motors (which convert chemical energy directly into kinetic energy), and Brownian motion with positive drift (which requires an external driving force to maintain the average motion of the fluid medium). Drift was not considered in our previous work. Secondly, our analysis in this paper uses much more sophisticated propagation models than that in our previous work. In particular, for Brownian motion, this paper simulates three-dimensional motion (versus two-dimensional motion in [8]), and for molecular motors, we use a more realistic model for vesicle loading of microtubules. Thus, our results move closer to a complete quantitative analysis of the microchannel molecular communication system.

\section{Simulation environment and models}

Our simulation environment is similar to the one in $[8,10]$. We use a rectangular propagation environment (with rounded corners), consisting of a strip loading zone and an unloading zone. Regardless of the propagation model, messagebearing vesicles originate at the loading zone, and propagate until they arrive at the unloading zone. If passive transport is used, information carrying vesicles follow a Brownian motion path from the loading zone to the unloading zone. If active transport is employed, mictotubule filaments moving over molecular motor tracks that cover the whole environment, pick up and transport the information carrying vesicles from the loading zone to the unloading zone. The reader is referred to [10] for detailed explanation of this process.

For Brownian motion, we perform a three-dimensional discrete-time simulation of information carrying vesicles, based on a simulation scheme from [11]. Given some initial position $\left(x_{0}, y_{0}, z_{0}\right)$ at time $t=0$, for any integer $k>0$, the motion of the vesicle is given by the sequence of coordinates $\left(x_{i}, y_{i}, z_{i}\right)$ for $i=1,2, \ldots, k$. Each coordinate $\left(x_{i}, y_{i}, z_{i}\right)$ represents the position of the particle at the end of time $t=i \Delta t$, where

$$
\begin{aligned}
x_{i} & =x_{i-1}+v_{f_{x}} \Delta t+\Delta r \cos \theta_{i} \cos \phi_{i}, \\
y_{i} & =y_{i-1}+v_{f_{y}} \Delta t+\Delta r \sin \theta_{i} \cos \phi_{i}, \\
z_{i} & =z_{i-1}+v_{f_{z}} \Delta t+\Delta r \sin \phi_{i},
\end{aligned}
$$

where $v_{f_{x}}, v_{f_{y}}$, and $v_{f_{z}}$ are flow velocity components in the $x, y$, and $z$ direction. Further, over each time interval of $\Delta t$, the molecule's displacement $\Delta r$ is given by

$$
\Delta r=\sqrt{4 D \Delta t},
$$

where $D$ is the free diffusion coefficient. For a given molecule and fluid propagation environment, $D$ is given by

$$
D=\frac{k_{B} T}{6 \pi \eta R_{H}}
$$

where $k_{B}=1.38 \cdot 10^{-23} \mathrm{~J} / \mathrm{K}$ is the Boltzman constant, $T$ is the temperature (in $\mathrm{K}$ ), $\eta$ is the dynamic viscosity of the fluid, and $R_{H}$ is the hydraulic radius 
of the molecule. We assume that $D$ is the same throughout the medium, and that collisions with the boundaries are elastic. In [11], values of $D$ ranging from $1-10 \mu \mathrm{m}^{2} / \mathrm{s}$ were considered realistic for signalling molecules. The angle, $\theta_{i}$ is an independent, identically distributed (iid) random variable for all $i$, uniformly distributed on $[0,2 \pi)$. The angle $\phi_{i}$ is also a iid random variable, uniformly distributed on $[-\pi / 2, \pi / 2)$. Figure 1 shows a sample simulation run.
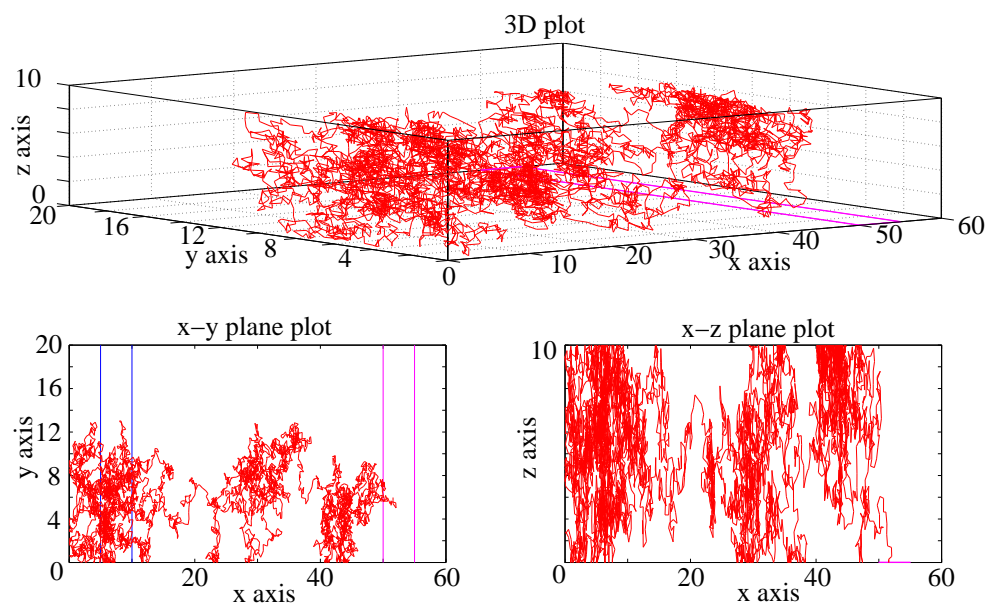

Fig. 1. A sample Brownian movement of a vesicle in three dimensions.

For molecular motors, as in [10], we assume that the microchannel is lined with static kinesin motors, and that these motors cause microtubule filaments to propagate along their surface. The motion of the microtubule is largely regular, although the effects of Brownian motion cause random fluctuations. We use a simulation scheme from [12], noting that microtubules stick to the motor-lined surface, so a two-dimensional discrete-time simulation is sufficient. In this case,

$$
\begin{aligned}
x_{i} & =x_{i-1}+\Delta r \cos \theta_{i}, \\
y_{i} & =y_{i-1}+\Delta r \sin \theta_{i} .
\end{aligned}
$$

In this case, the step size $\Delta r$ is an iid Gaussian random variable with mean $v_{\text {avg }} \Delta t$ and variance $2 D \Delta t$, where $v_{\text {avg }}$ is the average velocity of the microtubule, and $D$ is the microtubule's diffusion coefficient. The angle $\theta_{i}$ is no longer independent from step to step: instead, for some step-to-step angular change $\Delta \theta$, we have that

$$
\theta_{i}=\Delta \theta+\theta_{i-1} .
$$

Now, for each step, $\Delta \theta$ is an iid Gaussian-distributed random variable with mean $E[\Delta \theta]=0$ and variance $\operatorname{Var}[\Delta \theta]=v_{\text {avg }} \Delta t / L_{p}$, where $L_{p}$ is the persistence length of the microtubule's trajectory. In [12], these values were given as $v_{\text {avg }}=0.85$ 
$\mu \mathrm{m} / \mathrm{s}, D=2.0 \cdot 10^{-3} \mu \mathrm{m}^{2} / \mathrm{s}$, and $L_{p}=111 \mu \mathrm{m}$. Following [12], in case of a collision with a boundary, we assume that the microtubule sets $\theta_{i}$ so as to follow the boundary.

The loading and unloading mechanics are assumed to be the same as those proposed in [10]. Initialization, loading, and unloading have certain differences between Brownian motion and the molecular motors. We briefly outline the differences.

For Brownian motion, the vesicles start randomly and uniformly on the $(x, y)$ plane of the loading zone, but $z$ is set to the maximum vertical height (i.e., the molecule is "dropped" onto the microchannel). Furthermore, the vesicles start moving in Brownian motion as soon as they appear in the microchannel, so there is no need for loading. The propagation halts ("unloading") as soon as the vesicle arrives at the unloading zone.

For molecular motors, the vesicles are attached to the loading zone and do not move until they are picked up by a microtubule filament. The starting location of the microtubule is assumed to be random and uniformly distributed across the entire propagation area. Moreover, the initial directional angle $\theta_{0}$ is selected uniformly at random from the range $[0,2 \pi]$, and microtubules are assumed to be initially unloaded.

In order to capture the loading effect of active transport, in our simulations we divide the loading zone into a square grid, where the length of each square in the grid is the same as the diameter of the vesicles. We then distribute vesicle randomly and uniformly between the squares in the grid. If a microtubule enters a square which is occupied by a vesicle, we assume the microtubule loads that vesicle given it has an empty loading slot available. In general, we assume that the microtubules can load multiple vesicle and the maximum number of vesicles a microtubule can load is given by half of its length divided by the diameter of the vesicles. For unloading we assume all the loaded vesicles are unloaded as soon as a microtubule enters the unloading zone. Figure 2 shows a sample trajectory with the loading and unloading mechanism.

\section{Simulation Results}

We consider a microchannel with a width of $20 \mu \mathrm{m}$, a length of $60 \mu \mathrm{m}$, and height of $10 \mu \mathrm{m}$, as shown in the figure. The width of the loading strip and the unloading strip are assumed to be $5 \mu \mathrm{m}$ each. The diameter of the information carrying vesicles are assumed to $1 \mu \mathrm{m}$ and as the results the loading strip is divided into squares of $1 \mu \mathrm{m}$ length forming a grid over the loading zone. In case of the Brownian motion, the hight of the unloading zone is assumed to be $23 \mathrm{~nm}$ since an unloading mechanism based on ssDNAs is considered.

Throughout our simulations, we use $\Delta t=0.1 \mathrm{~s}$. For Brownian motion, that the $x$ axis is along the length of the microchannel, we set $v_{y}=v_{z}=0$, and use various values of $v_{x}$. either $v_{x}=0.3 \mu \mathrm{m} / \mathrm{s}$, or zero (for comparison); we also use $D=1 \mu \mathrm{m}^{2} / \mathrm{s}$. For molecular motors, we consider only a single microtubule 


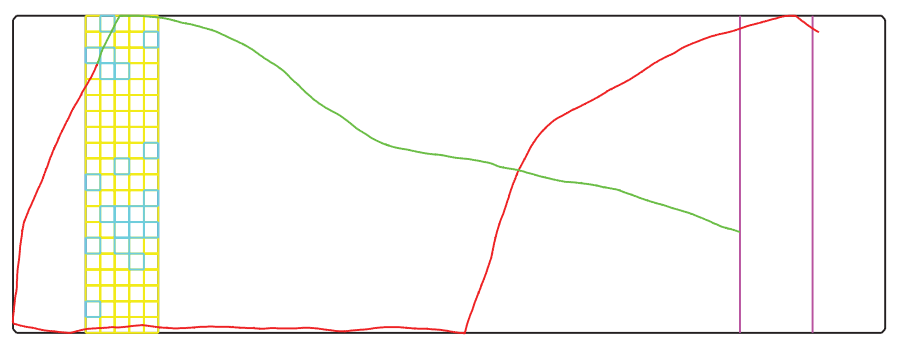

Fig. 2. A sample trajectory of active transport. The microtubule initially starts to the right of the unloading zone (box on the right side of the microchannel), and moves to the left (red trajectory). It passes through the loading zone (grid with empty yellow tiles and vesicle-bearing blue tiles) without encountering a vesicle, then passes through again, loading a vesicle. The loaded microtubule (green trajectory) then travels toward the unloading zone, where the trajectory terminates.

(which may transport up to five vesicles at once), using the parameters given in Section 2. Performance should improve as the number of microtubules increases.

We use the Blahut-Arimoto algorithm to optimize the input distribution of vesicles, which is upper bounded by the parameter $X_{\max }$. Figure 3 shows the mutual information versus maximum number of vesicles at the loading zone for time duration of 1000 seconds. As we can see introducing a small flow increases the information rate of Brownian motion significantly. However, for higher values of flow (e.g., $v_{x}=0.3$ ), performance declines; given our setup, this is because the vesicle speeds past the unloading zone. Overall Brownian motion with flow achieves a much higher information rate than both simple Brownian motion and active transport using a single microtubule.

\section{References}

1. S. Hiyama and Y. Moritani, "Molecular communication: Harnessing biochemical materials to engineer biomimetic communication systems," Nano Communicaiton Networks, vol. 1, pp. 20-30, 2010.

2. Y. Moritani, S. Hiyama, and T. Suda, "Molecular communication for health care applications," in Proc. 4th IEEE Intl. Conf. on Pervasive Computing and Communications Workshops, Pisa, Italy, 2006.

3. A. W. Eckford, "Nanoscale communication with Brownian motion," in Proc. Conf. on Information Sciences and Systems, Baltimore, MD, pp. 160-165, 2007.

4. B. Atakan and O. Akan, "An information theoretical approach for molecular communication," in Proc. 2nd Intl. Conf. on Bio-Inspired Models of Network, Information, and Computing Systems, Budapest, Hungary, 2007.

5. M. J. Moore, T. Suda, and K. Oiwa, "Molecular communication: Modeling noise effects on information rate," IEEE Trans. Nanobiosci., vol. 8, pp. 169-179, Jun. 2009.

6. A. W. Eckford, "Timing information rates for active transport molecular communication," in Proc. 4th Intl. Conf. on Nano-Networks, Luzern, Switzerland, pp. $24-28,2009$. 


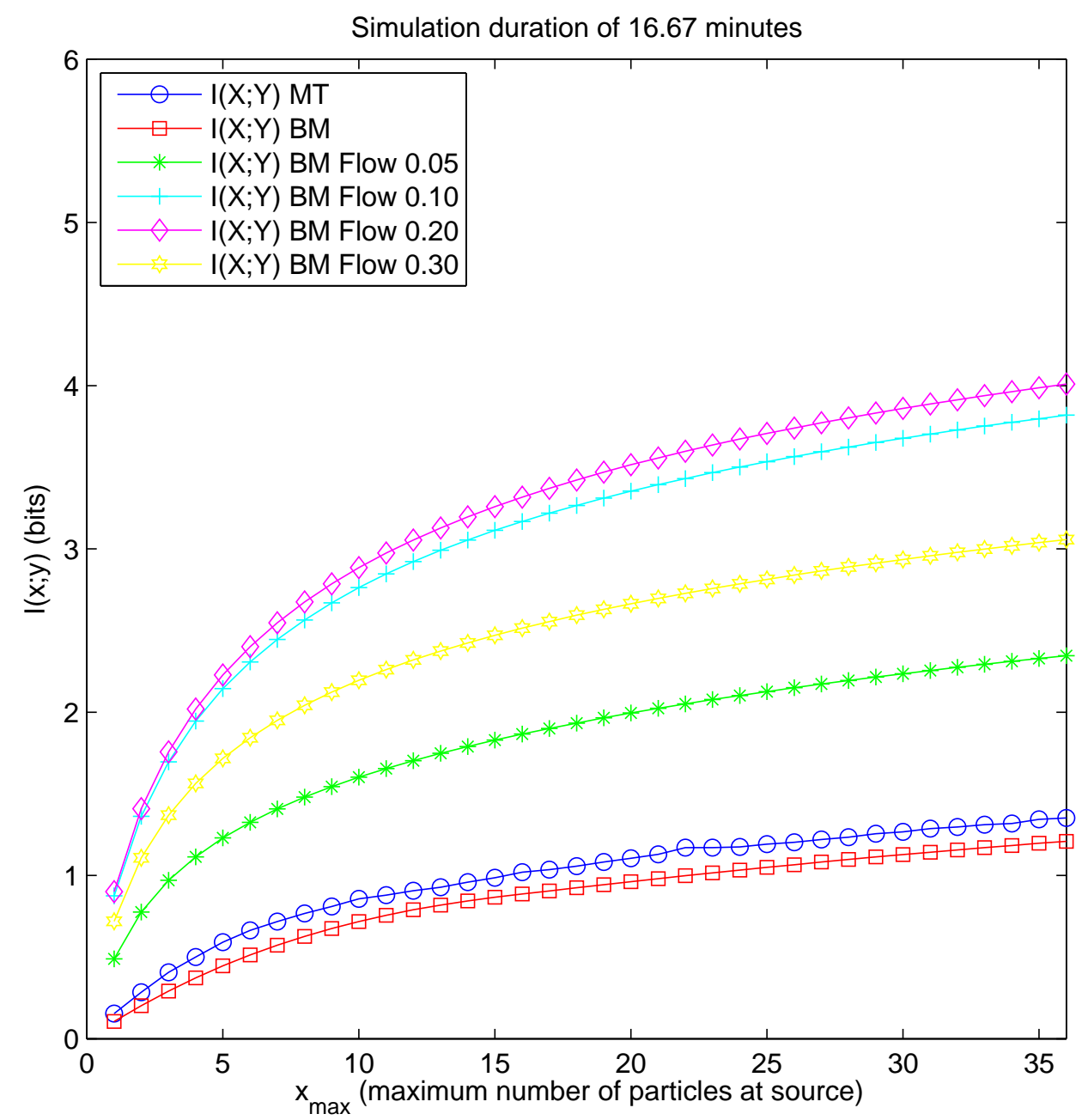

Fig. 3. Mutual information versus maximum number of vesicles at loading zone for time duration of 1000 seconds $(16 \mathrm{~m} \mathrm{40s).} \mathrm{Abbreviations} \mathrm{in} \mathrm{the} \mathrm{legend:} \mathrm{Microtubule}$ (MT); Brownian motion (BM); Brownian motion with flow (BM Flow, at the given velocity).

7. M. Pierobon and I. F. Akyildiz, "A physical end-to-end model for molecular communication in nanonetworks," IEEE J. Sel. Areas in Commun., vol. 28, no. 4, pp. 602-611, May 2010.

8. A. W. Eckford et al., "Microchannel molecular communication with nanoscale carriers: Brownian motion versus active transport," in IEEE Intl. Conf. on Nanotechnology, Seoul, South Korea, 2010.

9. T. M. Cover and J. A. Thomas, Elements of Information Theory, 2nd ed., Hoboken: Wiley, 2006. 
10. S. Hiyama et al., "Biomolecular-motor-based nano- or microscale particle translocations on DNA microarrays," Nano Lett., vol. 9, no. 6, pp. 2407-2413, Jun. 2009.

11. J. Berthier, Microfluidics for Biotechnology, Boston: Artech House, 2006.

12. T. Nitta et al., "Simulating molecular shuttle movements: Towards computer-aided design of nanoscale transport systems," Lab on a Chip, vol. 6, pp. 881-885, 2006. 